\title{
Point-of-care CD4+ technology implementation in Free State, South Africa, was associated with improved patient health outcomes
}

\author{
L van Turha, ${ }^{1}$ BN; K Maharaj, ${ }^{2}$ PGDBM; A Rose, ${ }^{3}$ PhD, MMed (Public Health); C Boeke, ${ }^{2}$ ScD; T F Peter, ${ }^{2}$ PhD; L Vojnov, ${ }^{2}$ PhD; \\ J Quevedo, ${ }^{2}$ BSc; Y Tsibolane, ${ }^{1}$ BA Cur \\ ${ }^{1}$ Free State Department of Health, Bloemfontein, South Africa \\ ${ }^{2}$ Clinton Health Access Initiative, Pretoria, South Africa \\ ${ }^{3}$ Department of Community Health, Faculty of Health Sciences, University of the Free State, Bloemfontein, South Africa
}

Corresponding author: Y Tsibolane (tsibolay@fshealth.gov.za)

Background. Point-of-care (POC) $\mathrm{CD} 4+$ technologies have the potential to increase patient access to treatment and care through rapid testing and result delivery at or close to where patients seek care. South African (SA) guidelines suggest the use of CD4+ testing to prioritise patients most in need of antiretroviral therapy (ART) and to support identification of patients with advanced HIV disease and opportunistic management of patients on ART. Understanding the patient impact of implementing POC CD4+ testing in the intended setting and operated by lower cadres of healthcare worker or non-professional healthcare facility staff will provide valuable insight into the appropriate use and placement of POC CD4+ technologies throughout SA.

Objectives. To determine the patient impact (turnaround time of tests, loss to follow-up, and proportions of eligible patients proceeding to the next steps in the testing and treatment cascade) of implementing POC CD4+ testing technologies compared with conventional laboratory-based CD4+ testing.

Methods. This retrospective cohort study included all HIV-positive adults from 30 healthcare facilities in Free State Province, SA. Healthcare facilities were placed into two groups (POC and laboratory referral) using a stratified randomisation technique based on the presence of a POC CD4+ technology and minimal ART volumes. Patients who received a CD4+ test prior to ART initiation between September 2012 and September 2014 were included. Data were collected from patient charts and the POC devices.

Results. For new patients, the average time from HIV diagnosis and CD4+ testing was reduced from 7.6 days in the laboratory referral group to 4.5 days in the POC group, a decrease of almost $60 \%$. Additionally, $59.6 \%$ of patients in the POC group received their HIV diagnosis and CD4+ test result on the same day, compared with $37.5 \%$ in the laboratory referral group (risk ratio (RR) 1.49 ; $95 \%$ confidence interval (CI) 1.01 - 2.18). Fewer patients were lost between HIV diagnosis and CD4+ testing (2.7\% v. 8.6\%) (RR 0.02; 95\% CI 0.05 - 0.78 ) in the POC group. The average test error rate across the study time period was $8.4 \%$; however, the error rate remained $<5 \%$ for the final 5 months of the study.

Conclusions. Introduction of the Alere Pima POC CD4+ technology in the Free State, operated by nurses and lay counsellors, was associated with positive patient outcomes across all parameters analysed. While this study highlighted an effective conventional laboratory network, a full costing and affordability analysis coupled with patient impact and access data from this study will provide further insight into the potential deployment strategies of POC CD4+ technologies in SA.

S Afr Med J 2020;110(2):126-131. https://doi.org/10.7196/SAMJ.2020.v110i2.13823

CD4+ testing is the most predictable indicator of HIV disease progression, and owing to limited resources is widely used to determine whether a patient is eligible for antiretroviral therapy (ART). Currently, in settings where it is available, CD4+ testing is used to determine whether a patient is eligible for ART, and it is more reliable in determining ART eligibility than symptomatic staging. ${ }^{[1,2]}$ Patients who do not have reliable on-site access to testing must often make numerous visits to healthcare facilities for testing blood draws, receiving test results, and routine clinical care visits due to sample and result transportation to and from centralised testing facilities. Furthermore, it has been suggested that up to $80 \%$ of patients are lost to follow-up before ART initiation. ${ }^{[3]}$

To overcome some of the challenges associated with centralised laboratory testing and patient loss to follow-up, high-quality pointof-care (POC) diagnostic technologies are available to alleviate critical testing needs in facilities lacking on-site $\mathrm{CD} 4+$ testing and provide same-day test results. ${ }^{[4]}$ Firstly, POC CD4+ technologies have the potential to increase patient access to $\mathrm{CD} 4+$ testing by reducing test turnaround time, allowing for more immediate clinical decisions, reducing patient loss to follow-up, and increasing the number of patients initiating ART. ${ }^{[5,6]}$ Secondly, POC technologies can reduce the burden of managing an extensive sample transportation network that cannot fully meet patient need and a growing network of conventional instruments that are increasingly difficult to maintain. Thirdly, providing POC testing administered by personnel with minimal technical training can make remote healthcare facilities more self-sufficient and allow for efficient task-shifting of testing and related tasks. ${ }^{[7]}$

\section{Objectives}

In 2012, the Free State Department of Health in South Africa (SA) implemented the Alere Pima POC CD4+ technology at 30 healthcare facilities. These technologies are used exclusively to determine ART 
eligibility of HIV-positive patients and are typically operated by nurses and lay counsellors. The primary objective and research question of this study was to determine the patient impact (turnaround time of tests, loss to follow-up, and proportions of eligible patients proceeding to the next steps in the testing and treatment cascade) of implementing POC CD4+ testing technologies compared with conventional laboratory-based CD4+ testing. Assessing the potential patient impact of POC CD4+ testing in the intended healthcare facility setting operated by lower cadres of healthcare facility staff will provide valuable insight into the appropriate and possible placement and use of POC CD4+ technologies throughout SA.

\section{Methods \\ Study setting}

Healthcare facilities with and without POC CD4+ were randomly placed into two groups using a stratified randomisation technique. A total of 235 publicly supported Department of Health facilities were considered for selection. To ensure that each healthcare facility included contributed relatively similar proportions of patient data, we stratified the random facility selection to include only those healthcare facilities with $>100$ pre-ART patients during the previous year. All 30 healthcare facilities with Pima technology were among the 135 healthcare facilities meeting these volumes. Fifteen of the 30 healthcare facilities with Pima technology were randomly selected to make up the POC group. Fifteen of the remaining 105 healthcare facilities without Pima technology were randomly selected to make up the laboratory referral group. Healthcare facilities in both groups were widely distributed throughout the province, covering 14 of the 19 municipalities.

\section{Study design}

This was a retrospective cohort study reviewing data from all HIVpositive adults aged $\geq 18$ years who received a CD4+ test prior to ART initiation from each of the 30 selected healthcare facilities. The Alere Pima POC CD4+ technology implementation began in March 2012 and was completed in the Free State in September 2012; only patients with CD4+ tests after September 2012 in both groups were therefore included. Data collection was conducted in September 2014 with data included from the previous 2 years for both groups. Patient charts were the primary tools used for data collection, while CD4+ testing registers and ART registers were also consulted. Sex, age, clinic visit dates, CD4+ test result and ART initiation status were captured for each patient. No personal identifiers were collected from patients. Records with inaccurate dates were corrected upon confirmation from the healthcare facility or excluded from analysis. In addition, primary raw data from the Alere Pima devices were downloaded when available to find out the percentage and types of errors encountered by healthcare facilities in the POC group as well as daily control bead tests run and operator volumes and errors. Errors can be due to operator or device malfunctions and are fatal, therefore requiring a repeat test. Internal control bead cartridges, consisting of one low CD4+ count and one normal CD4+ count cartridge, should be run each day prior to patient testing.

\section{Data analysis}

Primary parameters measured included retention and loss to followup along the testing and treatment cascade as well as time between steps in the testing and treatment cascade. Medians (interquartile ranges (IQRs)) and percentages were used for descriptive comparisons of values. Retention and loss-to-follow-up parameters were analysed using a univariate logistic model to estimate the risk ratios (RRs) and $95 \%$ confidence intervals (CIs). Regression coefficients were estimated using generalised estimating equations, accounting for patient clustering by healthcare facility. Robust standard errors were used to allow for different variances between groups. We used the exchangeable working correlation matrix, which assumes equal correlations between all clusters. A log link function and the Poisson distribution were used with a contrast statement to directly estimate RRs. Not all patients had complete records; retention along the testing and treatment cascade was therefore calculated using patient records that contained all necessary data for the specific metric. If a required date or information was absent, the patient was excluded from that specific analysis.

Patients were considered lost to follow-up if they: (i) did not receive their $\mathrm{CD} 4+$ test result and did not return to the healthcare facility within 90 days; (ii) did not initiate ART (if eligible) and did not return to the healthcare facility within 90 days; or (iii) did not return to the healthcare facility within 90 days.

The SA ART guidelines changed in the middle of this study and were incorporated into the analysis. The ART eligibility criteria changed from a threshold of 200 cells $/ \mu \mathrm{L}$ to 350 cells $/ \mu \mathrm{L}$ in March 2013. During the study period, patients were considered eligible for ART initiation with an absolute CD4+ count $\leq 200$ cells/ $\mu \mathrm{L}$ before March 2013 and $\leq 350$ cells $/ \mu$ after March 2013.

Data were entered into Excel version 16.16.15 (Microsoft Corp., USA) and analysed using SAS version 9.3 (SAS Institute, USA) and GraphPad Prism version 6.0 (GraphPad Software Inc., USA). Two researchers independently analysed and confirmed the results. The protocol for the study was reviewed and approved by the Ethics Committee of the University of the Free State and the Free State Department of Health and received a protocol exemption from the Chesapeake IRB (USA) (ref. no. Pro00010543).

\section{Results}

\section{Study demographics}

A total of 1491 patients were included in the study. Overall, 66.5\% of the patients were female (Table 1). Approximately $50 \%$ of enrolled patients were between the ages of 30 and 45 years, while the median (IQR) age was $35(29-43)$ years. The median CD4+ count was 314 cells/ $\mu \mathrm{L}$ and the median CD4+ count at ART initiation 243 cells/ $\mu \mathrm{L}$. Of patients with World Health Organization (WHO) staging documented $(n=1153)$, almost $70 \%$ were WHO stage 1 or 2 at presentation for $\mathrm{CD} 4+$ testing. In the POC group, the median $\mathrm{CD} 4+$ count was $327(184-504)$ cells $/ \mu \mathrm{L})$, while the median CD4+ count at ART initiation for eligible patients was $240(125-343)$ cells/ $\mu \mathrm{L}$. In the laboratory referral group, the median CD4+ count was 298 (152 - 463) cells $/ \mu \mathrm{L}$, while the median CD4+ count at ART initiation for eligible patients was $245(115$ - 342) cells $/ \mu \mathrm{L}$. These demographic results were not significantly different between the two groups. Approximately $52 \%$ of patients in the POC group and $59 \%$ of patients in the laboratory referral group were eligible for ART initiation (Fig. 1).

\section{Patient impact outcomes}

The median times between HIV diagnosis, CD4+ sample taken, CD4+ test completed and result received were $\leq 1$ day for both groups, with no significant differences between the groups. The proportion of patients who received their CD4+ test result was $>95 \%$ in both groups; however, more patients in the POC group than in the laboratory referral group received their result on the day of testing (83.5\% v. 64.1\%; RR 1.25; 95\% CI $1.01-1.54$; $p=0.04$ ) (Fig. 2A).

Comparable or improved patient impact was observed in the POC group compared with the laboratory referral group across most parameters analysed (Fig. 2B). Approximately $60 \%$ of patients 
Table 1. Study patient demographics

\begin{tabular}{|c|c|c|c|}
\hline & Total & POC & Laboratory referral \\
\hline All patients enrolled, $N$ & 1491 & 811 & 680 \\
\hline Female, \% & 66.5 & 67.3 & 65.6 \\
\hline Age (years), median (IQR) & $35(29-43)$ & $35(29-43)$ & $35(29-44)$ \\
\hline Age 30 - 45 years, $\%$ & 50.7 & 52.0 & 49.1 \\
\hline \multicolumn{4}{|l|}{ WHO stage, $\%$} \\
\hline 1 & 43.0 & 42.0 & 44.1 \\
\hline 2 & 25.8 & 28.6 & 22.7 \\
\hline 3 & 27.4 & 26.3 & 28.7 \\
\hline 4 & 3.8 & 3.2 & 4.5 \\
\hline CD $4+$ count $($ cells $/ \mu \mathrm{L})$, median (IQR) & $314(171-488)$ & $327(184-504)$ & $298(152-463)$ \\
\hline CD4+ count $($ cells $/ \mu \mathrm{L})$ at ART initiation, median (IQR) & $243(119-343)$ & $240(125-343)$ & $245(115-342)$ \\
\hline
\end{tabular}

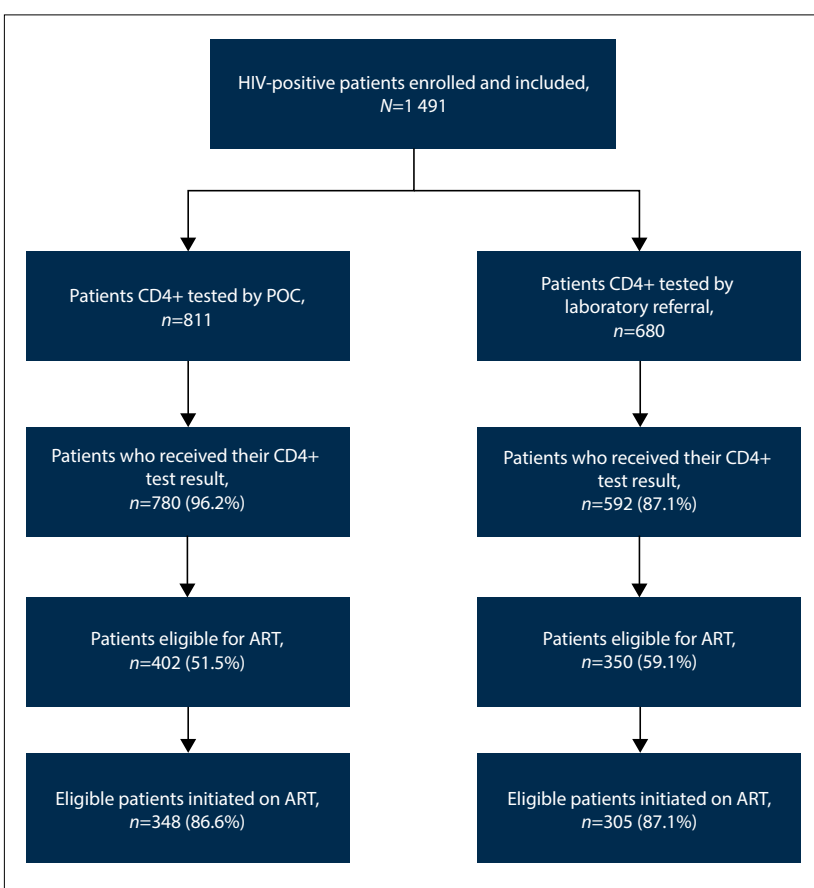

Fig. 1. Flow chart of retention along the testing and treatment cascade from $C D 4+$ testing to $A R T$ initiation. $(A R T=$ antiretroviral therapy; $P O C=$ point of care.)

received their HIV diagnosis and CD4+ test result on the same day in the POC group compared with $<40 \%$ in the laboratory referral group (RR 1.49; 95\% CI $1.01-2.18 ; p=0.04$ ). There was no significant difference in the proportion of eligible patients initiated on ART between the two groups. When focusing only on ART-eligible patients, we found that more patients received their $\mathrm{CD} 4+$ test result and were retained on ART for at least 6 months in the POC group than in the laboratory referral group (Fig. 2C).

Higher proportions of patients were retained in the testing and treatment cascade when tested by POC compared with laboratory referral (Fig. 2B). Loss to follow-up was significantly higher before $\mathrm{CD} 4+$ test results were received in the laboratory referral group compared with the POC group (8.6\% v. 2.7\%; RR 0.20; 95\% CI 0.05 $0.78 ; p=0.02$ ). Although higher proportions of patients in the laboratory referral group were lost to follow-up between determining ART eligibility and initiation, as well as after ART initiation, results were not statistically significant.
We next wanted to find out whether the observed changes in patient impact outcomes were due to early excitement about and attention to introducing a new technology to the healthcare facilities. Data were separated between the 2 years of the study for both groups, October 2012 - September 2013 and October 2013 - September 2014. For the POC group, although few samples were tested, eligible, and included in the first year, most patient impact parameters remained the same or improved in the second year of testing (data not shown).

\section{Quality control of the POC CD4+ technology}

The total Pima error rate for all healthcare facilities providing primary device data was $8.43 \%$. The most prevalent errors observed were invalid test error 850 (25.2\%), 880 (12.7\%) and 910 (10.9\%) (Fig. 3A). These three error types accounted for almost $50 \%$ of all errors. Manufacturer guidance suggests that invalid test error 850 and 880 can be either operator or equipment related, whereas invalid test error 910 is most often operator-related. Seventy percent of healthcare facilities had error rates $<10 \%$ for the total time period analysed. Interestingly, the five highest-volume healthcare facilities had error rates $<10 \%$ (Fig. 3B).

Although these error rates were higher than the minimal manufacturer-suggested error rate threshold of $5 \%$, we investigated whether performance improved over time. When the Pima technology is initially implemented, before healthcare facility staff become familiar with the technology, error rates can be higher. Indeed, when we analysed the data across time we saw that error rates dropped progressively. Since September 2013, error rates have remained at or below the minimal error rate threshold of 5\% (Fig. 3C). Four months were excluded from this analysis when healthcare facilities experienced stock-outs and were unable to test patients.

We also reviewed the regularity of running both the low and normal daily internal controls. Five out of the 13 healthcare facilities with data consistently ran both low and normal controls on $<95 \%$ of testing days. The remaining eight healthcare facilities ran both the low and normal controls on $<30 \%$ of testing days. Interestingly, however, 10 out of the 13 healthcare facilities ran at least one control on $>80 \%$ of patient testing days.

Healthcare cadres operating the POC CD4+ technology The POC CD4+ devices were placed in the ART clinics rather than in facility laboratories and were operated primarily by counsellors or nurses (Fig. 4). Counsellors conducted $\sim 70 \%$ of all Pima tests and nurses $\sim 15 \%$. Although counsellors and professional nurses 
A
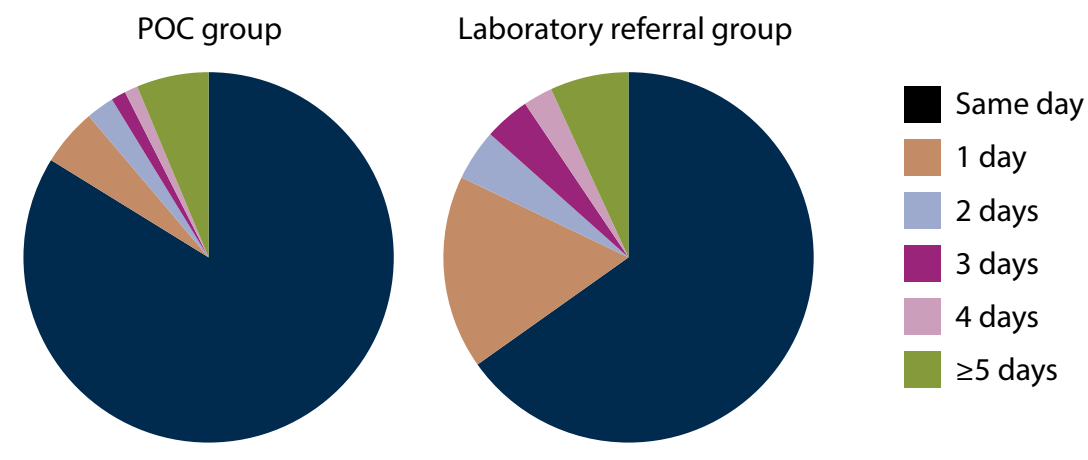

B

$\mathrm{RR}(95 \% \mathrm{Cl})$

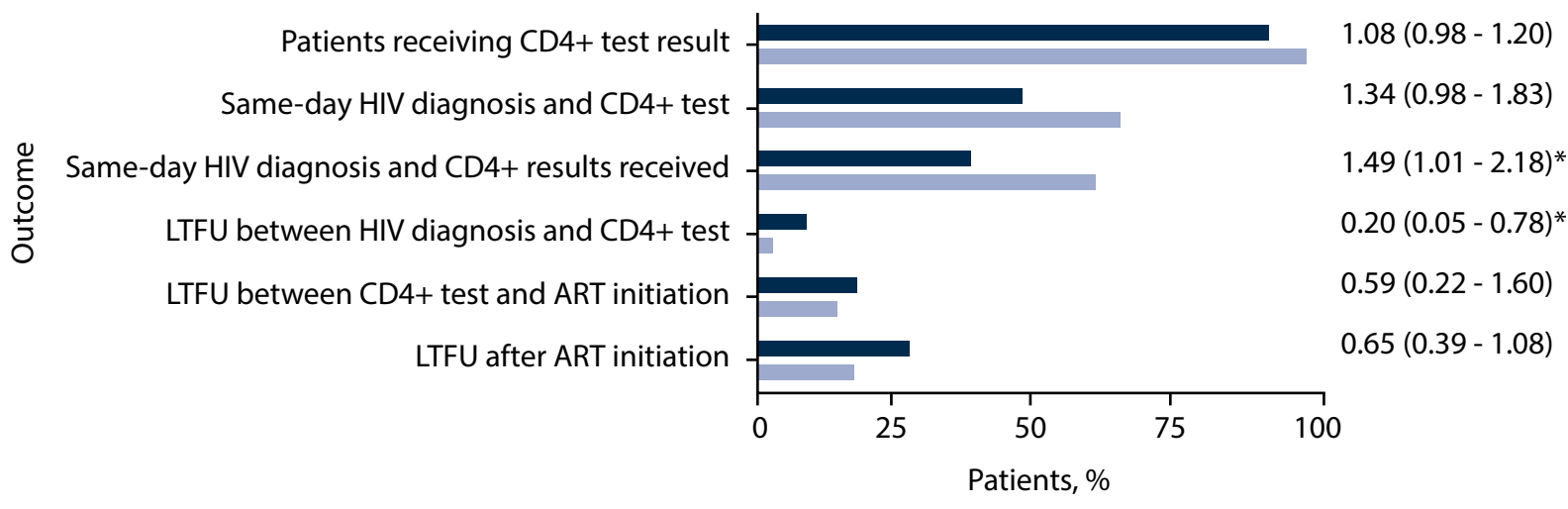

Laboratory referral group

POC group

\section{C}

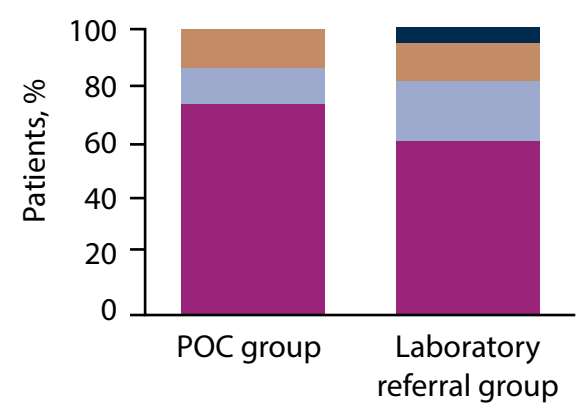

CD4+ tested

CD4+ result received

Initiated on ART

Retained on ART

Fig. 2. Patient impact outcomes: (A) distribution of turnaround times between CD4+ testing and result received for each group; (B) proportion of patients retained or lost along the testing and treatment cascade; and $(C)$ proportion of ART-eligible patients retained for at least 6 months along the testing and treatment cascade. $\left(P O C=\right.$ point of care; $L T F U=$ lost to follow-up; $A R T=$ antiretroviral therapy; $R R=$ risk ratio; $C I=$ confidence interval; ${ }^{*} \mathrm{p}<0.05$.

conducted $>85 \%$ of all Pima tests, their combined error rate was $<7.5 \%$. The error rate for counsellors was $6.8 \%$. Of healthcare cadres that conducted $>100$ tests, registered nurses had the highest error rate of $10.1 \%$.

\section{Discussion}

Introduction of the Alere Pima POC CD4+ technology in the Free State was associated with positive patient outcomes across all parameters analysed. Patients received their CD4+ test result earlier than they did when using the conventional referral network. A higher proportion of patients received a CD4+ test result, and a higher proportion of ART-eligible patients initiated ART. In addition, a lower proportion of patients were lost to follow-up across all time periods considered, including between HIV diagnosis and CD4+ test, between CD4+ test and ART initiation, and overall throughout the study. Although POC CD4+ on-site testing improved 
A

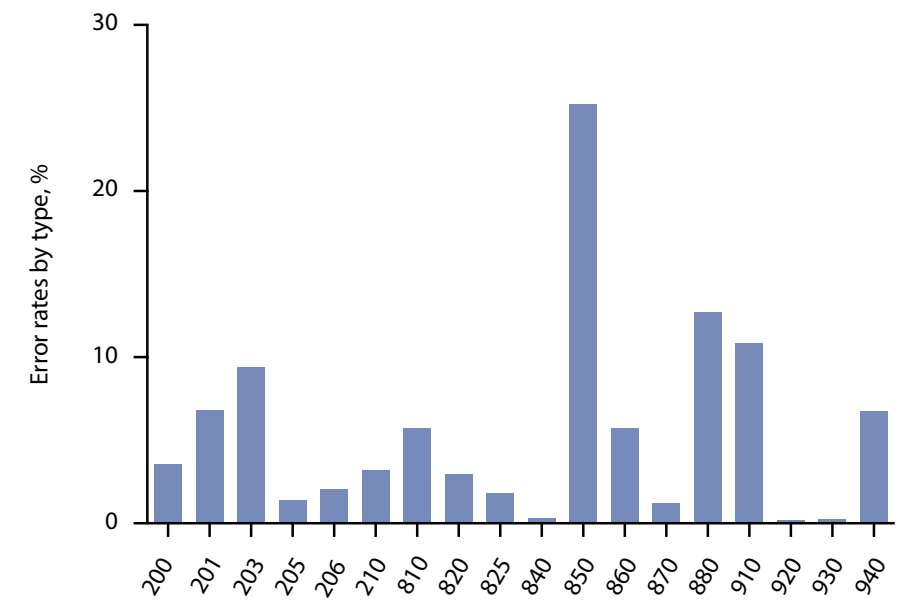

Manufacturer-designated error type

B

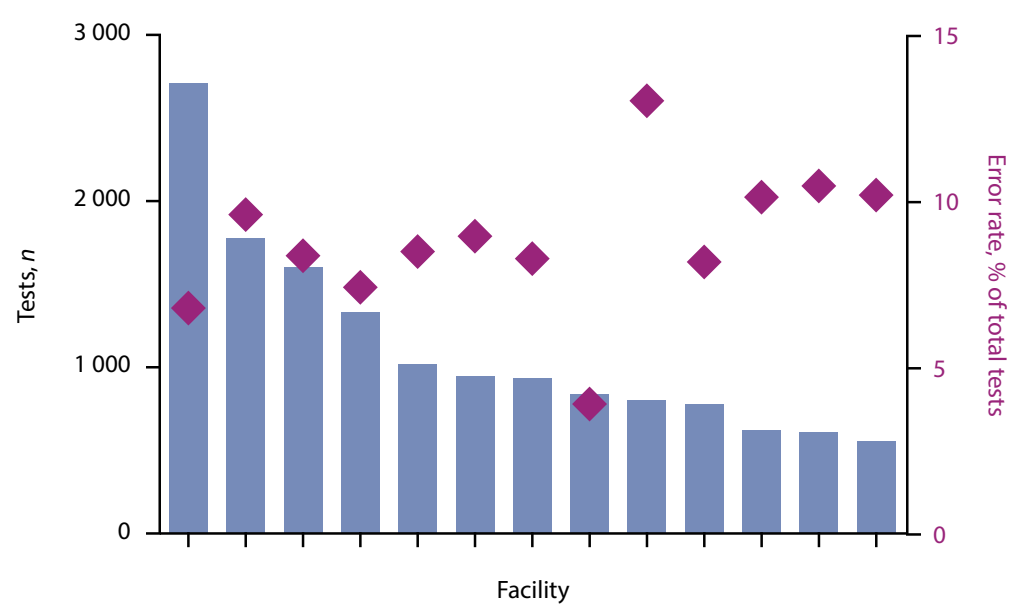

C

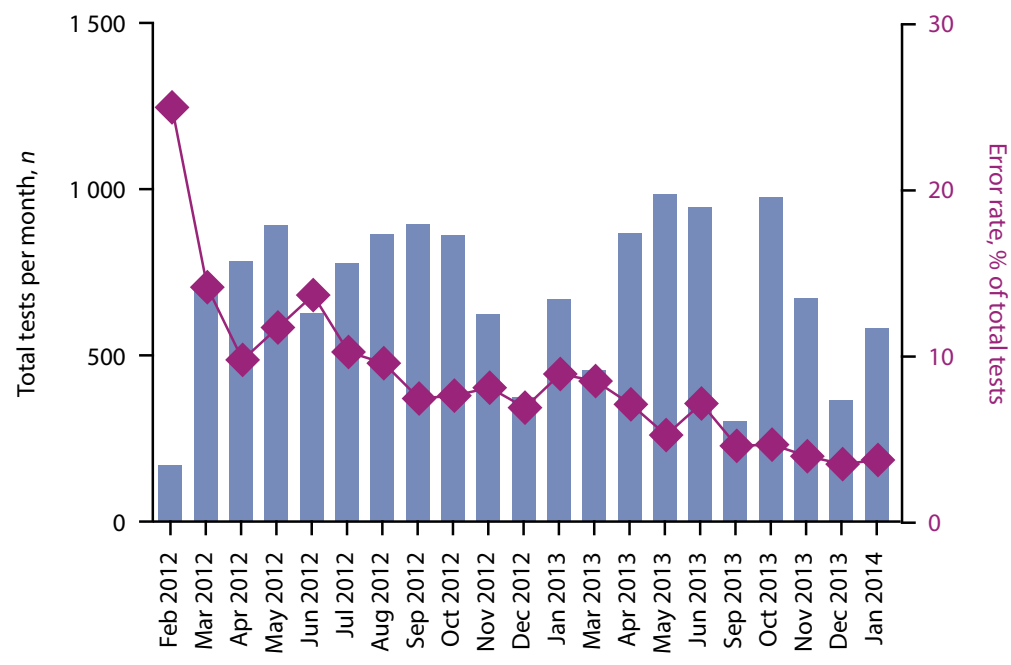

Fig. 3. Testing volumes and error rates by type (A), by facility (B) and over time (C). The purple diamonds in $B$ and $C$ represent the percentage error rate, while the blue bars represent testing volumes. outcomes, it is important to note that the performance of the conventional referral network provided good access to testing, with lower turnaround times and improved patient impact parameters compared with conventional referral networks observed in studies outside SA. ${ }^{[8-11]}$

The WHO recently released recommendations suggesting the uptake of 'treat all' for all HIV-positive patients. ${ }^{[12]}$ As countries clinically, logistically and financially consider implementing 'treat all' widely, POC CD4+ may help to prioritise patients in most need of ART, particularly in settings with unpredictable drug supplies. Furthermore, SA guidelines suggest the use of CD4+ testing at ART initiation and to support HIV disease and opportunistic infection management of patients on ART. POC testing may allow for wider decentralisation of HIV care, expedited clinical decisions and self-sufficient facilities, thus further improving the health system. ${ }^{[7]}$

While it is worth noting that the present work was an evaluation of programmatic implementation of POC CD4+ and not designed as a study, the results were consistent with previous studies conducted in SA. One study found that patients with access to POC CD4+ were more likely to return for further care (RR 1.25). ${ }^{[13]}$ Additionally, two randomised controlled trials found that ART-eligible patients who received a POC CD4+ test were significantly more likely to initiate ART than those who received a laboratory-based CD4+ test. ${ }^{[14,15]}$ Rosen et al. ${ }^{[15]}$ also found that earlier ART initiation in patients using POC CD4+ did not result in greater attrition after initiation compared with the standard of care. Finally, similar time reductions were observed between HIV diagnosis, CD4+ staging and ART initiation. ${ }^{[14,16]}$ The present study highlights that the previous research findings are consistent and applicable in a provincial public implementation programme.

The results observed are not as significant as we expected, or very different compared with results from other countries. ${ }^{[9]}$ This is probably due to an optimised and efficient CD4+ sample collection and result delivery system established by the National Health Laboratory Service as well as strong national infrastructure to allow for swift transport of samples. Furthermore, many of the healthcare facilities in the laboratory referral group were within $100 \mathrm{~km}$ of their reference CD4+ laboratory. In fact, 7 of the 15 healthcare facilities in the laboratory referral group were within $25 \mathrm{~km}$ of their reference CD4+ laboratory. It would there- 


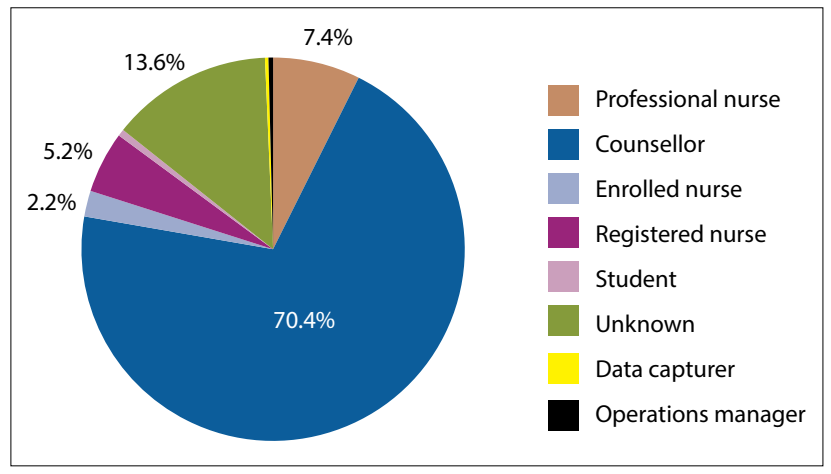

Fig. 4. Proportion of tests conducted by different operator cadres.

fore be worthwhile to find out whether the impact of POC CD4+ testing would be more significant in rural healthcare facilities further from their reference CD4+ laboratory.

While this and other studies clearly emphasise superior patient impact performance of POC CD4+ technologies compared with conventional laboratory-based testing, the cost-effectiveness and affordability of implementing this programme in the light of new guidelines are unclear. POC CD4+ has been shown to be costeffective in Mozambique; ${ }^{[17]}$ however, no similar study has been conducted in the SA context. A conventional and POC tiered approach has been suggested, ${ }^{[18,19]}$ but the costs associated with both testing types are not sufficiently clear to indicate the affordability of each scenario in SA. Additional cost-effectiveness, costing and modelling work would be beneficial to determine the implications of POC CD4+ implementation into the national network in SA.

\section{Study limitations}

This study has several limitations. It was an observational retrospective programmatic implementation evaluation and therefore lacked the strength of a randomised controlled trial. In designing the study, we sought to limit sources of bias through randomly selecting facilities, consecutively enrolling all patients who matched the inclusion criteria, and maintaining the same time periods for both groups. Additional confounders may have occurred that were not incorporated into the final analysis. Fortunately, data management was strong and patient records were typically complete.

\section{Conclusions}

POC CD4+ improves timeliness and linkage to ART, but POC technologies are not the solution to all systems challenges. Although error rates were reduced over time to $<5 \%$, refresher training and routine monitoring would improve the testing system. Furthermore, the POC CD4+ technologies were not enrolled in any quality assurance programme for proficiency testing or monitored remotely using connectivity. Additional strengthening of the supply chain network, service and maintenance, and clinical guidance could further increase the observed patient impact. Implementation of POC CD4+ technologies will support faster, high-quality test result delivery and linkage in SA, while future research highlighting the patient impact and cost-effectiveness of POC CD4+ technologies in the context of advanced HIV disease identification would be beneficial.

\section{Declaration. None.}

Acknowledgements. We thank the staff at each of the included 30 healthcare facilities for their dedication to this work. We also thank Freshly Ground Insights for their efficient and complete data collection.

Author contributions. LvT, YT: designed the study, interpreted results, wrote the first draft of the manuscript, and approved the final version. KM, LV: designed the study, performed the analysis, interpreted results, wrote the first draft of the manuscript, developed figures and tables, and produced the final version. AR, TFP, JQ: designed the study, substantially commented on manuscript drafts, and approved the final version. CB: performed the analysis, interpreted results, and approved the final version of the manuscript.

Funding. We acknowledge funding from the Department for International Development (UK).

Conflicts of interest. None.

1. Hulgan T, Shepherd BE, Raffanti SP, et al. Absolute count and percentage of CD4+ lymphocytes are independent predictors of disease progression in HIV-infected persons initiating highly active antiretroviral therapy. J Infect Dis 2007;195(3):425-431. https://doi.org/10.1086/510536

2. Kagaayi J, Makumbi F, Nakigozi G, et al. WHO HIV clinical staging or CD 4 cell counts for antiretroviral 2. Kagaayi J, Makumbi F, Nakigozi G, et al. WHO HIV clinical staging or CD4 cell counts for antiretroviral
therapy eligibility assessment? An evaluation in rural Rakai district, Uganda. AIDS 2007;21(9):12081210. https://doi.org/10.1097/QAD.0b013e32810c8dce

3. Rosen S, Fox M. Retention in HIV care between testing and treatment in sub-Saharan Africa: A systematic review. PLoS Med 2011;8(7): e1001056. https://doi.org/10.1371/journal.pmed.1001056 4. UNITAID, World Health Organization. HIV/AIDS Diagnostic Technology Landscape. 3rd ed. 2013. https://aidsfree.usaid.gov/resources/prevention-update/editions/july-2013/hivaids-diagnostictechnology-landscape-third (accessed 13 December 2019).

5. Vojnov L, Markby J, Boeke C, Harris L, Ford N, Peter T. POC CD4 testing improves linkage to HIV care and timeliness of ART initiation in a public health approach: A systematic review and meta-analysis. PLoS One 2016;11(5):e0155256. https://doi.org/10.1371/journal.pone.0155256

6. Wynberg E, Cooke G, Shroufi A, Reid SD, Ford N. Impact of point-of-care CD4 testing on linkage to HIV care: A systematic review. J Int AIDS Soc 2014;17:18809. https://doi.org/10.7448/IAS.17.1.18809 . Jani IV, Peter TF. How point-of-care testing could drive innovation in global health. N Engl J Med 2013;368(24): 2319-2324. https://doi.org/10.1056/NEJMsb1214197

8. Brouillette AM, Chien E, Nyegenye W, Tenywa T, Grosz J. Using finger-prick blood collection samples for the Pima POC CD4 device increases access to CD4 testing (Abstract WEPE607). Presented at the
forillette 7th IAS Conference on HIV Pathogenesis and Treatment, Kuala Lumpur, Malaysia, 30 June - 3 July 2013.

9. Jani IV, Sitoe NE, Alfai ER, et al. Effect of point-of-care $\mathrm{CD} 4$ cell count tests on retention of patients and rates of antiretroviral therapy initiation in primary health clinics: An observational cohort study. Lancet 2011;378(9802):1572-1579. https://doi.org/10.1016/S0140-6736(11)61052-0

10. Mwanja A, Brown L, Mtumbuka E, Shayo C, Vojnov L. The introduction of a POC CD4 technology increases patient access to and the impact of CD4 testing (Abstract WEPE608). Presented at the 7th IAS Conference on HIV Pathogenesis and Treatment, Kuala Lumpur, Malaysia, 30 June - 3 July 2013.

11. Rioja MR, Nzuobontane D, Munyaburanga F, et al. CD4 count testing capacity in rural health districts in Cameroon through point-of-care technology (Abstract No WEPE609). Presented at the 7th IAS Conference on HIV Pathogenesis and Treatment, Kuala Lumpur, Malaysia, 30 June - 3 July 2013.

12. World Health Organization (2015) Guideline on when to start antiretroviral therapy and on preWorld Health Organization (2015) Guideline on when to start antiretroviral therapy and on pre-
exposure prophylaxis for HIV. https://www.who.int/hiv/pub/guidelines/earlyrelease-arv/en/ (accessed 6 December 2019).

13. Larson B, Schnippel K, Ndibongo B, et al. Rapid point-of-care CD4 testing at mobile HIV testing sites to increase linkage to care. J Acquir Immune Defic Syndr 2012;61(2):e13-e17. https://doi.org/10.1097/ QAI.0b013e31825eec60

14. Faal M, Naidoo N, Glencross DK, Venter WD, Osih R. Providing immediate CD4 count results at HIV testing improves ART initiation. J Acquir Immune Defic Syndr 2011;58(3):e54-e59. https://doi. org/10.1097/QAI.0b013e3182303921

15. Rosen S, Maskew M, Fox MP, et al. Rapid ART initiation reduces loss between HIV testing and treatment: The RapIT Trial (Abstract 1091). 2015 Conference on Retroviruses and Opportunistic Infections, Seattle, USA, 23 - 26 February 2015. https://www.croiconference.org/scientific-program/ electronic-materials/croi-2015 (accessed 13 December 2019).

16. Patten GE, Wilkinson L, Conradie K, et al. Impact on ART initiation of point-of-care CD4 testing at HIV diagnosis among HIV-positive youth in Khayelitsha, South Africa. J Int AIDS Soc 2013;16:18518. https://doi.org/10.7448/IAS.16.1.18518

17. Hyle EP, Jani IV, Lehe J, et al. The clinical and economic impact of point-of-care CD4 testing in Mozambique and other resource-limited settings: A cost-effectiveness analysis. PLoS Med 2014;11(9):e1001725. https://doi.org/10.1371/journal.pmed.1001725

18. Cassim N, Coetzee LM, Schnippel K, Glencross DK. Estimating implementation and operational costs of an integrated tiered CD4 service including laboratory and point of care testing in a remote health
and district in South Africa. PLoS One 2014;9(12):e115420. https://doi.org/10.1371/journal.pone.0115420 19. Glencross DK, Coetzee LM, Cassim N. An integrated tiered service delivery model (ITSDM) based on local CD4 testing demands can improve turn-around times and save costs whilst ensuring accessible and scalable CD4 services across a national programme. PLoS One 2014;9(12):e114727. https://doi. org/10.1371/journal.pone.0114727 